\title{
Evaluation of Therapeutic Effects of Astaxanthin on Impairments in Salivary Secretion
}

\author{
Takashi Yamada ${ }^{1}$, Koufuchi Ryo ${ }^{1}$, Yoshinori Tai ${ }^{2}$, Yoh Tamaki ${ }^{3}$, Hiroko Inoue ${ }^{1}, K$ Keni Mishima ${ }^{1}$, \\ Kazuo Tsubota ${ }^{4}$ and Ichiro Saito ${ }^{1, *}$ \\ ${ }^{1}$ Department of Pathology, Tsurumi University School of Dental Medicine, \\ 2-1-3, Tsurumi, Tsurumi-ku, Yokohama 230-8501, Japan \\ ${ }^{2}$ Department of Geriatric Dentistry, Tsurumi University School of Dental Medicine, \\ 2-1-3, Tsurumi, Tsurumi-ku, Yokohama 230-8501, Japan \\ ${ }^{3}$ Department of Policy Sciences, National Institute of Public Health, \\ 2-3-6 Minami, Wako-shi, Saitama 351-0197, Japan \\ ${ }^{4}$ Department of Ophthalmology, Keio University School of Medicine, \\ 35 Shinanomachi, Shinjuku-ku, Tokyo 160-8582, Japan
}

Received 11 March, 2010; Accepted 8 April, 2010; Published online 22 June, 2010

\begin{abstract}
Summary The involvement of reactive oxygen species (ROS) in the pathophysiology of Sjögren's syndrome (SS), an autoimmune disorder, and irradiation-induced impairments in salivary secretion has been reported. Meanwhile, the strong antioxidant astaxanthin (Ast) has been suggested to have therapeutic effects on various diseases. In the present study, we examined the ROS scavenging capacity of Ast using a human salivary gland epithelial cell line (HSY) and investigated the effects of Ast on salivary secretion in a mouse model of irradiation-induced salivary gland dysfunction. Furthermore, we performed a clinical study of Ast in six SS patients and six normal individuals, quantifying the volume of saliva secretion and the level of oxidative stress markers in the saliva. Ast partially suppressed hydrogen peroxide-induced ROS in HSY cells. The mouse model demonstrated that the preadministration of Ast resulted in the suppression of irradiation-induced hyposalivation. Furthermore, the administration of Ast appeared to increase salivary output in both the SS and normal groups. The level of oxidative stress marker, hexanoyl-lysine, in the saliva was reduced after Ast intake. These results suggest that Ast might act as an ROS scavenger, providing benefits to SS patients with impaired salivary secretion.
\end{abstract}

Key Words: astaxanthin, sjögren's syndrome, salivary secretion, reactive oxygen species

\section{Introduction}

Sjögren's syndrome (SS) is an autoimmune disorder characterized by lymphocyte infiltration and the destruction of the lacrimal and salivary glands, accompanied by the systemic production of autoantibodies to the ribonucleo-

\footnotetext{
*To whom correspondence should be addressed

Tel: +81-45-580-8360 Fax: +81-45-572-2888

E-mail: saito-i@tsurumi-u.ac.jp
}

protein particles SS-A/Ro and SS-B/La. The disease is not fatal, but the dysfunction of the exocrine glands causes the hyposecretion of tears and saliva, resulting in dry eyes and dry mouth. These are common problems among the elderly, and living with SS can be a devastating experience for the sufferer, decreasing the quality of life dramatically. The causes of SS have not yet been clarified; however, the involvement of environmental factors and oxidative stress from reactive oxygen species (ROS) [1] as well as genetic factors, immune disorders, and viral infections has been suggested in the onset and pathology of SS [2]. In particular, 
ROS such as superoxide and hydrogen peroxide are known to induce apoptosis [3] and cause oxidative damage to membrane proteins and lipids, leading to impaired functioning [4]. Similar mechanisms have also been implicated in the pathology of several diseases $[5,6]$. Numerous reports have discussed possible mechanisms whereby various pathophysiological phenomena associated with autoimmune diseases develop through these processes [7,8]. For example, increased levels of oxidative stress markers have been found in the synovial fluid [9, 10], serum [11-13], and urine of patients with rheumatoid arthritis [14] and type I diabetes mellitus $[15,16]$.

Antioxidants are expected to serve as potentially therapeutic agents for oxidative stress-related diseases. Astaxanthin (Ast) is a reddish orange compound that belongs to the carotenoid family, which is widely found in fishes such as sea bream and salmon as well as crustaceans such as crab and shrimp. An important function of carotenoids such as Ast is to intercept the chlorophyll triplet state, in order to prevent the formation of singlet oxygen, or to quench the singlet oxygen molecule directly $[17,18]$. In recent years, the strong antioxidant action of Ast has been implicated in preventive mechanisms for many disorders [19, 20].

In this study, we examined the therapeutic effects of Ast on impairments in salivary secretion in a mouse model of irradiation-induced salivary hypofunction and SS patients with xerostomia. As part of a clinical study, a gumdrop containing Ast was administered to twelve subjects: six SS patients with xerostomia and six normal individuals. To investigate the capacity of Ast to scavenge intracellularly generated ROS, we used a human salivary gland epithelial cell line (HSY) and evaluated the amount of ROS generated by $\mathrm{H}_{2} \mathrm{O}_{2}$ using flow cytometry.

\section{Materials and Methods}

\section{Experimental animals}

Six-month-old male C57BL6J mice were purchased from Japan Clea Inc. (Tokyo, Japan) and maintained under specific pathogen-free conditions until use. All the experimental procedures were approved by the animal welfare committee of Tsurumi University (Kanagawa, Japan).

\section{Irradiation}

Each mouse was anesthetized using an intraperitoneal injection of $60 \mathrm{mg} / \mathrm{kg}$ sodium pentobarbital. A single acute exposure to a 10-MV $\mathrm{X}$ rays (MEVATRON74 DX40; Toshiba Medical Systems, Tokyo, Japan) at a dose rate of $3 \mathrm{~Gy} / \mathrm{min}$ and a distance of $1,000 \mathrm{~mm}$ was performed. The effective radiation dose to the salivary gland was set using the percentage depth dose and was over $95 \%$ of the maximum dose delivered.

\section{Administration of Ast}

The Ast was provided by Fuji Chemical Industry Co., Ltd (Toyama, Japan). To examine the preventive and therapeutic effects of Ast on impaired salivary secretion mediated by ROS, the mice were freely fed food containing 2, 10 or $50 \mathrm{mg}$ of Ast. In the model examining preventive effects, free feeding was performed beginning two weeks before irradiation and was continued until four weeks after irradiation in the model examining therapeutic effects, free feeding was performed for 4 weeks after irradiation.

\section{Measurement of salivary secretion}

The volume of saliva secreted by the Ast-treated mice was measured. The amount of saliva secretion was measured 1 week prior to irradiation and was used to classify the animals into administration groups with similar secretory capabilities. The mice were weighed and then anesthetized using an intraperitoneal injection of a mixture of xylazine $(24 \mathrm{mg} / \mathrm{kg})$ and ketamine $(36 \mathrm{mg} / \mathrm{kg})$. To stimulate salivation, pilocarpine $(0.1 \mathrm{mg} / \mathrm{kg})$ was injected intraperitoneally. The saliva secreted into the oral cavity during each 1-min period following the injection of pilocarpine was carefully collected using capillaries (Ringcaps; Hirschmann Laborgeräte $\mathrm{GmbH}$ \& Co. KG, Eberstadt, Germany). The amount of total saliva secreted in 15 min was divided by the weight of the mouse.

\section{Human salivary gland epithelial cell cultures}

HSY cells derived from an adenocarcinoma of the parotid gland were used. HSY cells were cultured in growth medium containing high-glucose Dulbecco's modified Eagle's medium (DMEM) (D6429; Sigma, St. Louis, MO) plus 10\% fetal bovine serum (Sigma, St. Louis, MO) and 1\% penicillin or streptomycin (Invitrogen, Carlsbad, CA) and maintained in a $100-\mathrm{mm}$ culture dish in a humidified atmosphere containing $5 \% \mathrm{CO}_{2}$ at $37^{\circ} \mathrm{C}$. Cells were allowed to grow to $80 \%$ confluence before being passaged in trypsin-EDTA solution. The cells were then reseeded in a $100-\mathrm{mm}$ culture dish and grown for $24 \mathrm{~h}$ before use in the experiments.

\section{Measurement of ROS using fluorescence-activated cell sorting}

We assessed ROS generation using the fluorescent probe CM- $\mathrm{H}_{2}$ DCFDA $(5 \mathrm{mM})$, a dye that is sensitive to a broad spectrum of ROS including $\mathrm{H}_{2} \mathrm{O}_{2}$ and $\mathrm{OH}$ [21]. CM$\mathrm{H}_{2}$ DCFDA is a cell permeant indicator for ROS that is nonfluorescent until the removal of the acetate groups by oxidation within the cell. Cells were grown at a density of $1 \times 10^{6}$ cells per well in a 12 -well plate and were maintained for $24 \mathrm{~h}$ in growth medium, then incubated for $1 \mathrm{~h}$ with Ast $(5 \mathrm{mM})$ or $\mathrm{N}$-acetyl-cysteine (NAC) $(5 \mathrm{mM})$ and exposed for $15 \mathrm{~min}$ to $\mathrm{CM}-\mathrm{H}_{2}$ DCFDA. The HSY cells were then exposed to $1.0 \mathrm{mM} \mathrm{H}_{2} \mathrm{O}_{2}$ for $30 \mathrm{~min}$ to analyze the intracellular ROS level. Analyses were performed using a 
Table 1. Characteristic of the subjects in this study

\begin{tabular}{lcr}
\hline & SS & Normal \\
& $(n=6)$ & $(n=6)$ \\
\hline age (years) & $64.7 \pm 6.4$ & $28.5 \pm 4.0$ \\
saliva flow rate $(\mathrm{g} / 2 \mathrm{~min})$ & $1.02 \pm 0.4$ & $6.2 \pm 2.2$ \\
\hline
\end{tabular}

Values represent mean \pm SEM.

SS: Sjögren syndrome

FACSCaliber flow cytometer and Cell Quest software (Becton Dickinson, Franklin Lakes, NJ).

Evaluation of Ast effects on SS patients and normal individuals

Tablets containing $2 \mathrm{mg}$ of Ast extracted from Haematococcus pluvialis were used (provided by Fuji Chemical Industry Co., Ltd. Kamiichi-machi, Japan). Six SS patients with xerostomia who had a salivary output of less than $2 \mathrm{~g}$, as determined using the Saxon test (SS group), and six normal individuals (normal group) were examined (Table 1). All twelve participants provided informed consent. Each participant took six test tablets per day $(12 \mathrm{mg} /$ day). Tests were performed before and 2 weeks after the oral intake of the Ast tablets. Participants were instructed not to eat food containing Ast during the examination period. A piece of sterilized gauze was weighed before and after being chewed by a participant for $2 \mathrm{~min}$. The difference between the two measurements (dry weight before chewing and wet weight after chewing) was regarded as the salivary output. Pre-treated saliva samples were centrifuged at $10,000 \mathrm{rpm}$ for $30 \mathrm{~min}$ and then passed through an ultrafiltration membrane (pore size, $0.22 \mu \mathrm{m}$ ). The filtered samples were subjected to an enzyme-linked immunosorbent assay for the measurement of the oxidative stress marker 8hydroxy-2'-deoxyguanosine $(8-\mathrm{OHdG})$ and the lipid peroxidation marker hexanoyl-lysine (HEL) using an anti-8OHdG monoclonal antibody (N45.1; Institute for the Control of Aging, Shizuoka, Japan) and an anti-HEL monoclonal antibody (Institute for the Control of Aging, Shizuoka, Japan), respectively. The primary antibodies bound to the markers were probed with an HRP-conjugated anti-mouse IgG antibody (Zymed Laboratories, South San Francisco, $\mathrm{CA}$ ) and assessed by measuring the color development at $490 \mathrm{~nm}$.

\section{Statistical analysis}

All the results were expressed as the mean \pm SE. The Wilcoxon's rank-sum test was performed to compare the results before and after the intake of the Ast tablets. Data were analyzed for statistical significance, and the significance level was set at a $p$ value $<0.05$.
Ethics

Informed consent was obtained from all the patients, and the Ethical Committee of Tsurumi University approved this study.

\section{Results}

Comparison of Ast effects on irradiation-induced impaired salivary secretion

The present study showed that salivary flow had decreased at 1 week after irradiation in the control group, which was fed a conventional chow (Fig. 1). This result is consistent with that of a previous report. The administration of a high dose of Ast ( $50 \mathrm{mg} /$ day) before irradiation resulted in the suppression of the salivary impairment observed in the control group at 3 and 4 weeks after irradiation (Fig. 1A). However, when Ast was administered after irradiation, the salivary secretion did not recover (Fig. 1B). These findings indicate that Ast has a preventive, but not a therapeutic, effect on irradiation-induced salivary dysfunction.

Confirmation of antioxidant activities of Ast and NAC using fluorescence-activated cell sorting flow cytometry

The intracellular generation of ROS at $20 \mathrm{~min}$ after the addition of $\mathrm{H}_{2} \mathrm{O}_{2}$ was measured using CM-H2DCFDA as a fluorescent indicator of ROS, such as $\mathrm{H}_{2} \mathrm{O}_{2}$ and hydroxyl radicals. The mean fluorescence intensity (MFI) of $\mathrm{H}_{2} \mathrm{O}_{2}$ stimulated HSY cells was elevated $(\mathrm{MFI}=485.04)$. Pretreatment with Ast partially suppressed the $\mathrm{H}_{2} \mathrm{O}_{2}$-induced MFI elevation $(\mathrm{MFI}=117.51)$. Although the lower-phase intensity was not changed, the higher-phase intensity was almost completely inhibited (Fig. 2B). This result suggests the possibility that Ast scavenges some species of ROS induced by $\mathrm{H}_{2} \mathrm{O}_{2}$. On the other hand, pre-treatment with NAC, scavenges wide variety of ROS including $\mathrm{H}_{2} \mathrm{O}_{2}$ and $\cdot \mathrm{OH}$ via glutathione generation [22], reverted the $\mathrm{H}_{2} \mathrm{O}_{2}$ induced MFI elevation to the unstimulated levels $(\mathrm{MFI}=$ 18.11) (Fig. 2A).

Effects of Ast on saliva secretion and oxidative stress markers in SS patients and normal individuals

The involvement of oxidative stress in salivary gland dysfunction has been reported [1]. When the SS group was compared with the normal group, the levels of both 8-OHdG and HEL were significantly higher in the SS group than in the normal group ( $p=0.008$ and $p=0.005$, respectively) (Fig. 3). This result was consistent with those of previous studies [1]. To evaluate the effects of Ast on salivary secretion and ROS production in SS patients, we measured the amount of salivary secretion and the level of 8-OHdG and HEL in saliva before and after examination. Although the increased amount of salivary secretion after the intake of Ast for 2 weeks was faint in the SS group (1.02 g/2 min to 
A

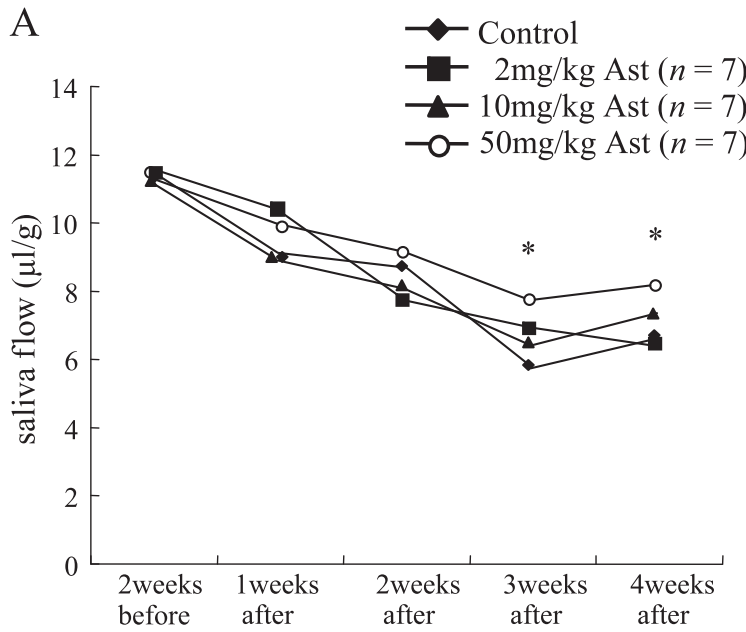

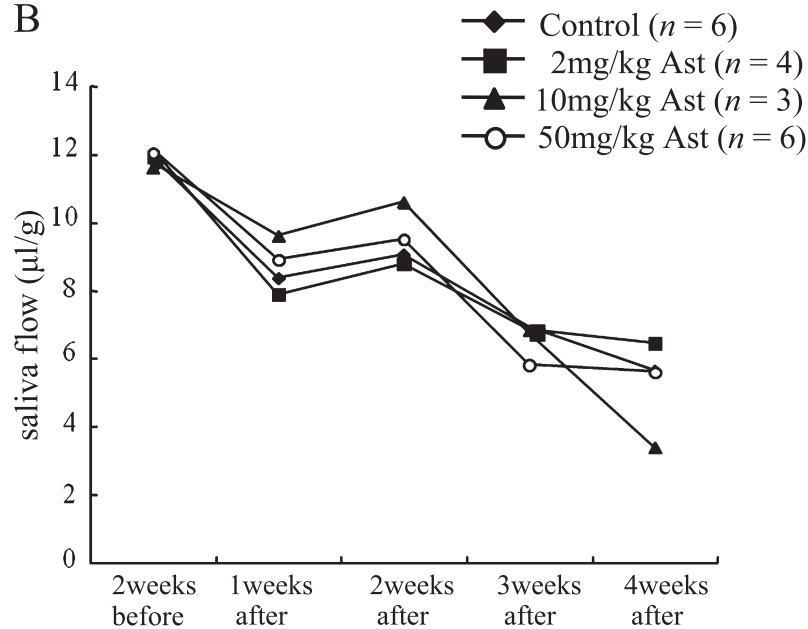

Fig. 1. Effect of Ast on salivary secretion. A) Model examining preventive effects. Ast at a dose of $2 \mathrm{mg} / \mathrm{kg}, 10 \mathrm{mg} / \mathrm{kg} \mathrm{or} 50 \mathrm{mg} / \mathrm{kg} \mathrm{was}$ administered intravenously 2 weeks prior to irradiation and once a day beginning on the day after irradiation and continuing for 4 weeks. B) Model examining therapeutic effects. Ast at a dose $2 \mathrm{mg} / \mathrm{kg}, 10 \mathrm{mg} / \mathrm{kg}$ or $50 \mathrm{mg} / \mathrm{kg}$ was administered intravenously after irradiation and once a day beginning on the day after irradiation and continuing for 4 weeks. Saliva flow was expressed as the total output of saliva during the first $15 \mathrm{~min}$ after pilocarpine stimulation, normalized to the body weight. The asterisks indicate a significant decrease $\left({ }^{*} p<0.05\right)$, compared with the respective control.

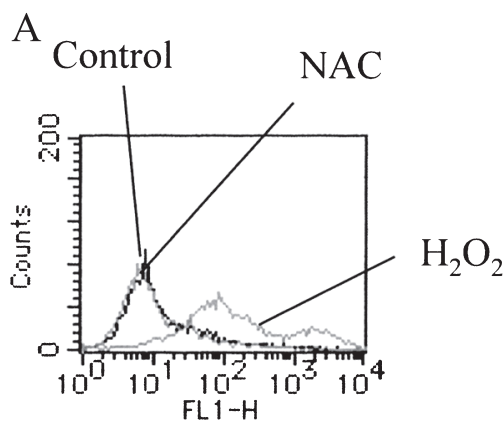

B

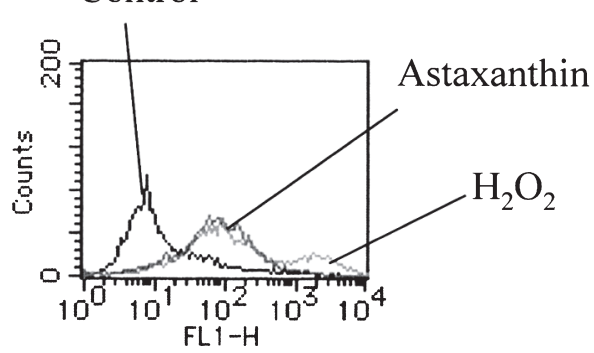

Fig. 2. Flow cytometry analysis of ROS. HSY cells were incubated for $1 \mathrm{~h}$ with NAC (5 mM) (A) or Ast (5 mM) (B) and then exposed for $15 \mathrm{~min}$ to $\mathrm{CM}-\mathrm{H}_{2} \mathrm{DCFDA}$. ROS generation was assessed after exposure to $1.0 \mathrm{mM}$ $\mathrm{H}_{2} \mathrm{O}_{2}$ for $30 \mathrm{~min}$.
$1.04 \mathrm{~g} / 2 \mathrm{~min}, p=0.69$ ) (Fig. 4A), a significant increase was observed in the normal group $(6.23 \mathrm{~g} / 2 \mathrm{~min}$ to $7.02 \mathrm{~g} / 2 \mathrm{~min}$, $p=0.03$ ) (Fig. 4B). The level of the oxidative stress marker 8-OHdG did not change in either group (Fig. 5, left panels of $\mathrm{A}$ and $\mathrm{B})$. However, the alternative oxidative stress marker HEL was significantly decreased in SS group $(34.0 \mathrm{M}$ to $30.58 \mathrm{M}, p=0.03$ ) (Fig. 5, right panel of A). The decreased HEL level in the normal group was faint (Fig. 5, right panel of B). This suggests that the elevated stress marker levels shown in this study were not caused by hyposalivation. These results suggest that Ast administration has a beneficial effect on salivation in not only patients with SS, but also in normal individuals by inducing the scavenging of ROS.

\section{Discussion}

The possible causes of hyposalivation include organic disorders of glandular tissues, as exemplified by the autoimmune disease SS, as well as the adverse effects of drugs, pollution, and aging. High levels of oxidative stress markers such as $8-\mathrm{OHdG}$ and HEL in the saliva of SS patients [1] and the involvement of ROS in the mechanism of irradiationinduced hyposalivation [23] have been reported. These findings suggest that the oxidative stress induced by various environmental factors causes salivary gland dysfunction [24]. Ast, a member of the carotenoid family, has been reported to have an extremely high level of antioxidant activity and to act as a scavenger of oxygen radicals [25]. For this reason, the clinical studies of Ast have been accumulated [26-28]. Satoh et al. showed the safety of 
A

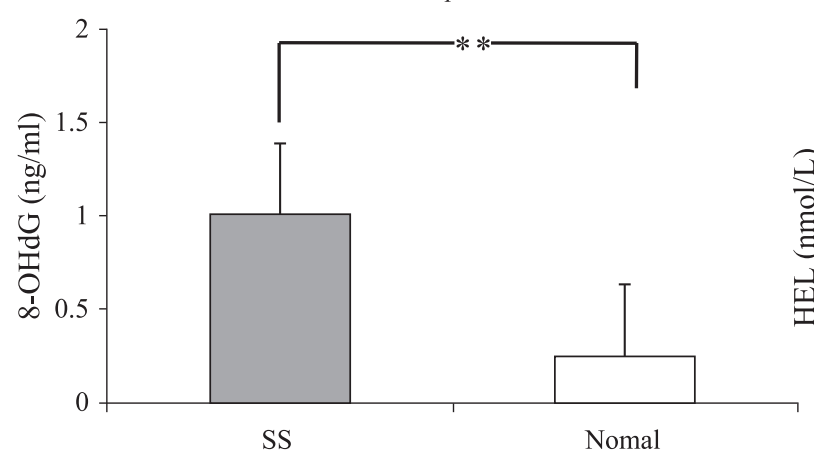

B

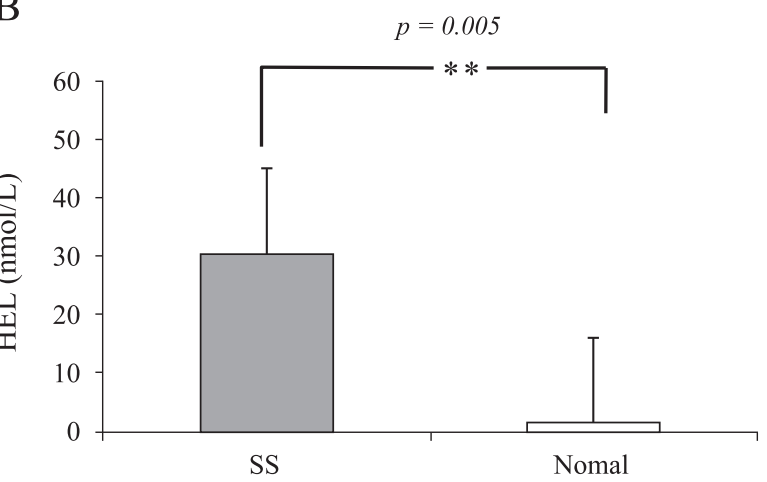

Fig. 3. 8 -OHdG $(p=0.008)$ and HEL $(p=0.005)$ levels of saliva in both groups. These levels were significantly higher in the SS group than in the normal group. The asterisks indicate significant increases $\left({ }^{* *} p<0.005\right)$. Values are the mean \pm SEM. A) SS group, B) normal group.

A

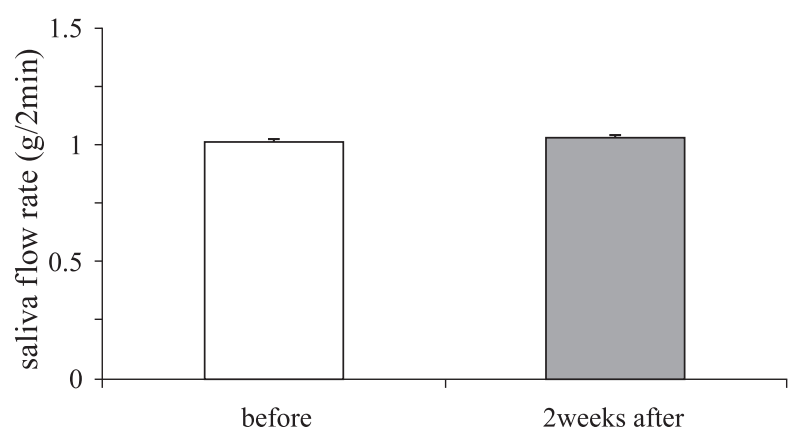

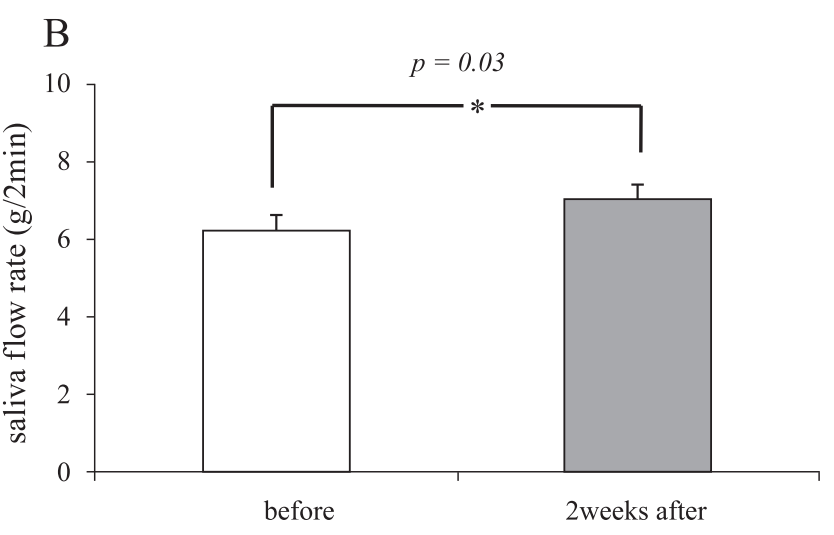

Fig. 4. Measurement of salivary output upon stimulation using the Saxon test. In the normal group treated with Ast, the saliva flow rate after 2 weeks of treatment was significantly higher than that at baseline. The asterisks indicate a significant increase $(* p<0.05)$. Values are the mean \pm SEM. A) SS group, B) normal group.

new Ast-product and the efficiency of Ast on age-related decline in cognitive and psychomotor functions. Therefore, we examined the effects of Ast on the ROS scavenging capacity and the possible therapeutic effect of Ast on salivary gland dysfunction. We found that the levels of the oxidative stress markers 8 -OHdG and HEL were significantly higher in the SS group than in the normal group (Fig. 3) and that the level of HEL in the SS group was significantly reduced by the intake of Ast (Fig. 5A). These results suggest that oxidative stress may be a cause of salivary gland dysfunction in SS. Although the Ast-induced inhibition of HEL in the SS group was higher than that in the normal group (Fig. 5), the effect of Ast on salivation was faint in the SS group but significant in the normal group (Fig. 4). These inconsistencies might have arisen for several reasons. First, the level of ROS production in the SS group might be too high to be compensated for by Ast under the study conditions, including the dose and/or term of treatment. Second, the species of ROS affecting salivary function might be generated as a byproduct of normal cellular respiration, associated primarily with mitochondrial electron transport [29]. In the SS group, in addition to the above mechanism, ROS might be generated following cell lysis and the oxidative burst produced by the immune response $[30,31]$. Therefore, the increase in salivation in the SS group was not as strong as expected.

Takeda et al. [23] reported that $\mathrm{N}^{\mathrm{G}}$-monomethyl-L-arginine (L-NMMA), a nitric oxide synthase inhibitor, alleviated hyposalivation in irradiated mice and inferred that nitrogen monoxide was involved in the impairment of salivary secretion. Based on these findings and the fact that Ast inhibits nitrogen monoxide synthesis [32], the alleviation of hyposalivation by Ast in an irradiation-induced hyposalivation mouse model shown in the present study (Fig. 1) might be due to a defense mechanism involving the inhibition of nitrogen monoxide synthesis. However, Ast did not show a therapeutic effect on irradiation-induced hyposalivation. Thus, the scavenging of ROS generated at the time of 
A

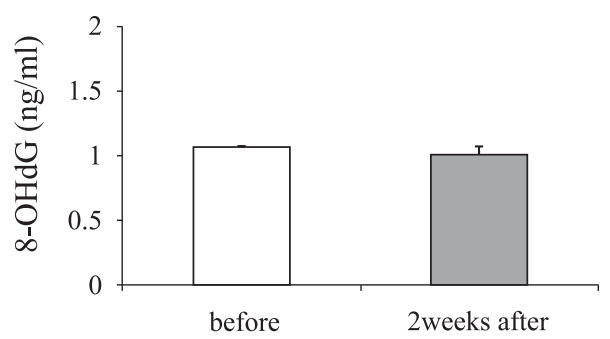

B

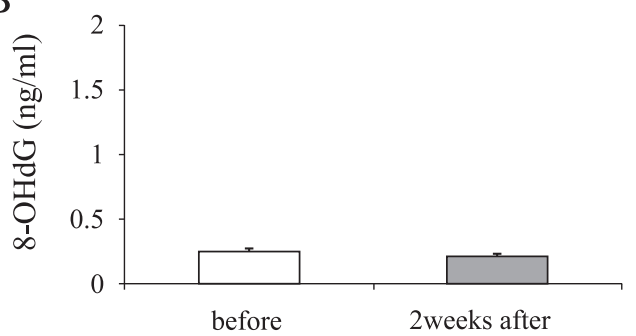

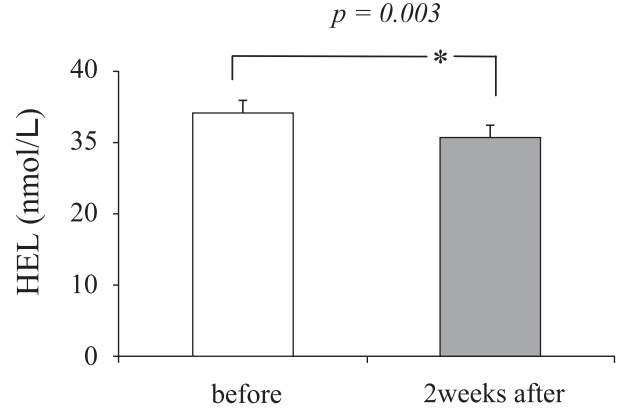

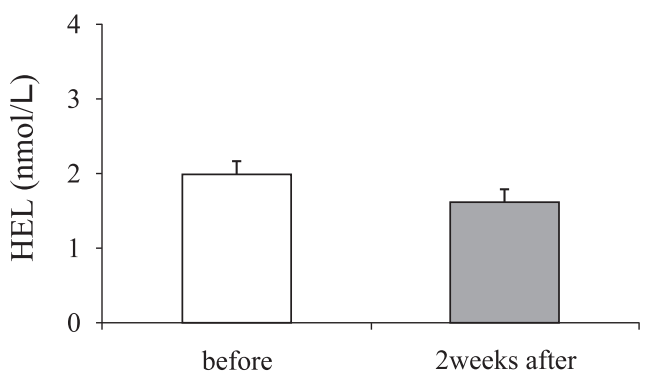

Fig. 5. Effect of Ast on 8-OHdG and HEL levels in saliva in both groups. In the SS group treated with Ast, the HEL level decreased significantly after treatment, compared with the baseline value. The asterisks indicate a significant decrease $\left({ }^{*} p<0.05\right)$. Values are the mean \pm SEM. A) SS group, B) normal group.

irradiation may be crucial. In the patients with SS, the reduction in HEL might have resulted from the Ast-induced scavenging of newly generated ROS, which accounts for a small portion of the total HEL in the saliva of patients with SS. Therefore, treatment with Ast might be useful in patients with salivary dysfunction associated with SS to prevent the condition from worsening.

The expression of various inflammatory cytokines including IL-1, IL-2, IFN $\gamma$ and TGF $\beta$, cell adhesion molecules, and chemokines is detected in salivary gland of SS patients [33-35]. In normal human mesangial cells (NHMCs), Ast suppresses high glucose induced ROS production. Accompanied with ROS production, Ast suppresses the activation of nuclear transcription factors such as nulclear factor kappa $\mathrm{B}(\mathrm{NF} \kappa \mathrm{B})$ and activator protein (AP-1), and the expression of transforming growth factorbeta 1 (TGF $\beta 1$ ), and monocyte chemoattractant protein-1 (MCP-1) [36]. Thus, Ast may be effective in the cytokine production following tissue destruction in the salivary gland of SS patients resulting in reduced salivary dysfunction.

Impairments in salivary secretion affect the quality of life and also cause various functional impairments, including dental caries, periodontal diseases, upper gastrointestinal disorders, aspiration pneumonia, dysphagia and infectious diseases. Therefore, the development of a medicine for impaired salivary secretion is of great significance, and Ast is a potent candidate for such a remedy. We plan to perform further clinical evaluations using a larger number of cases and to clarify the details of the mechanism of impaired salivary secretion in the future.

\section{Acknowledgments}

This work was partially supported by grants-in-aid for scientific research from the Ministry of Education, Culture, Sports Science and Technology of Japan. The authors gratefully thank Judith Nishino for helpful discussions during the preparation of this manuscript.

\section{Abbreviations}

ROS, reactive oxygen species; SS, Sjögren's syndrome; Ast, astaxanthin; HEL, hexanoyl-lysine; HSY, human salivary gland epithelial cell line; 8-OHdG, 8-hydroxy-2'deoxyguanosine.

\section{References}

[1] Ryo, K., Yamada, H., Nakagawa, Y., Tai, Y., Obara, K., Inoue, H., Mishima, K., and Saito, I.: Possible involvement of oxidative stress in salivary gland of patients with Sjogren's syndrome. Pathobiology, 73, 252-260, 2006.

[2] Fox, R.I.: Sjögren's syndrome. Lancet, 366, 321-331, 2005.

[3] Maheshwari, A., Misro, M.M., Aggarwal, A., Sharma, R.K., and Nandan, D.: Pathways involved in testicular germ cell apoptosis induced by $\mathrm{H}_{2} \mathrm{O}_{2}$ in vitro. FEBS J., 276, 870-881, 
2009.

[4] Dukan, S., Farewell, A., Ballesteros, M., Taddei, F., Radman, M., and Nyström, T.: Protein oxidation in response to increased transcriptional or translational errors. Proc. Natl. Acad. Sci. U.S.A., 97, 5746-5749, 2000.

[5] Houstis, N., Rosen, E.D., and Lander, E.S.: Reactive oxygen species have a causal role in multiple forms of insulin resistance. Nature, 440, 944-948, 2006.

[6] Babbar, N. and Casero, R.A. Jr.: Tumor necrosis factor-alpha increases reactive oxygen species by inducing spermine oxidase in human lung epithelial cells: a potential mechanism for inflammation-induced carcinogenesis. Cancer Res., 66, 11125-11130, 2006.

[7] Saito, I., Shimuta, M., Terauchi, K., Tsubota, K., Yodoi, J., and Miyasaka, N.: Increased expression of human thioredoxin/ adult T cell leukemia-derived factor in Sjögren's syndrome. Arthritis Rheum., 39, 773-782, 1996.

[8] Bashir, S., Harris, G., Denman, M.A., Blake, D.R., and Winyard, P.G.: Oxidative DNA damage and cellular sensitivity to oxidative stress in human autoimmune diseases. Ann. Rheum. Dis., 52, 659-666, 1993.

[9] Miyata, T., Ishiguro, N., Yasuda, Y., Ito, T., Nangaku, M., Iwata, H., and Kurokawa, K.: Increased pentosidine, an advanced glycation end product, in plasma and synovial fluid from patients with rheumatoid arthritis and its relation with inflammatory markers. Biochem. Biophys. Res. Commun., 244, 45-49, 1998.

[10] Mantle, D., Falkous, G., and Walker, D.: Quantification of protease activities in synovial fluid from rheumatoid and osteoarthritis cases: comparison with antioxidant and free radical damage markers. Clin. Chim. Acta, 284, 45-58, 1999.

[11] Ozkan, Y., Yardým-Akaydýn, S., Sepici, A., Keskin, E., Sepici, V., and Simsek, B.: Oxidative status in rheumatoid arthritis. Clin. Rheumatol., 26, 64-68, 2007.

[12] Baskol, G., Demir, H., Baskol, M., Kilic, E., Ates, F., Karakukcu, C., and Ustdal, M.: Investigation of protein oxidation and lipid peroxidation in patients with rheumatoid arthritis. Cell Biochem. Funct., 24, 307-311, 2006.

[13] Jikimoto, T., Nishikubo, Y., Koshiba, M., Kanagawa, S., Morinobu, S., Morinobu, A., Saura, R., Mizuno, K., Kondo, S., Toyokuni, S., Nakamura, H., Yodoi, J., and Kumagai, S.: Thioredoxin as a biomarker for oxidative stress in patients with rheumatoid arthritis. Mol. Immunol., 38, 765-772, 2002.

[14] Rall, L.C., Roubenoff, R., Meydani, S.N., Han, S.N., and Meydani, M.: Urinary 8-hydroxy-2'-deoxyguanosine (8$\mathrm{OHdG}$ ) as a marker of oxidative stress in rheumatoid arthritis and aging: effect of progressive resistance training. J. Nutr. Biochem., 11, 581-584, 2000.

[15] Reznick, A.Z., Shehadeh, N., Shafir, Y., and Nagler, R.M.: Free radicals related effects and antioxidants in saliva and serum of adolescents with Type 1 diabetes mellitus. Arch. Oral Biol., 51, 640-648, 2006.

[16] Hata, I., Kaji, M., Hirano, S., Shigematsu, Y., Tsukahara, H., and Mayumi, M.: Urinary oxidative stress markers in young patients with type 1 diabetes. Pediatr. Int., 48, 58-61, 2006.

[17] Tatsuzawa, H., Maruyama, T., Misawa, N., Fujimori, K., and Nakano, M.: Quenching of singlet oxygen by carotenoids produced in escherichia coli-attenuation of singlet oxygenmediated bacterial killing by carotenoids. FEBS Lett., $\mathbf{4 8 4}$, 280-284, 2000.

[18] Cantrell, A., McGarvey, D.J., Truscott, T.G., Rancan, F., and Böhm, F.: Singlet oxygen quenching by dietary carotenoids in a model membrane environment. Arch. Biochem. Biophys., 412, 47-54, 2003.

[19] Schroeder, W.A. and Johnson, E.A.: Singlet oxygen and peroxyl radicals regulate carotenoid biosynthesis in Phaffia rhodozyma. J. Biol. Chem., 270, 18374-18379, 1995.

[20] Ben-Dor, A., Steiner, M., Gheber, L., Danilenko, M., Dubi, N., Linnewiel, K., Zick, A., Sharoni, Y., and Levy, J.: Carotenoids activate the antioxidant response element transcription system. Mol. Cancer Ther., 4, 177-186, 2005.

[21] Wedgwood, S. and Black, S.M.: Induction of apoptosis in fetal pulmonary arterial smooth muscle cells by a combined superoxide dismutase/catalase mimetic. Am. J. Physiol. Lung Cell. Mol. Physiol., 285, L305-312, 2003.

[22] Aruoma, O.I., Halliwell, B., Hoey, B.M., and Butler, J.: The antioxidant action of $\mathrm{N}$-acetylcysteine: its reaction with hydrogen peroxide, hydroxyl radical, superoxide, and hypochlorous acid. Free Radic. Biol. Med., 6, 593-597, 1989.

[23] Takeda, I., Kizu, Y., Yoshitaka, O., Saito, I., and Yamane, G.Y: Possible role of nitric oxide in radiation-induced salivary gland dysfunction. Radiat. Res., 159, 465-470, 2003.

[24] Vitolo, J.M., Cotrim, A.P., Sowers, A.L., Russo, A., Wellner, R.B., Pillemer, S.R., Mitchell, J.B., and Baum, B.J.: The stable nitroxide tempol facilitates salivary gland protection during head and neck irradiation in a mouse model. Clin. Cancer Res., 10, 1807-1812, 2004.

[25] Kim, J.H., Choi, W., Lee, J.H., Jeon, S.J., Choi, Y.H., Kim, B.W., Chang, H.I., and Nam, S.W.: Astaxanthin inhibits $\mathrm{H}_{2} \mathrm{O}_{2}$-mediated apoptotic cell death in mouse neural progenitor cells via modulation of P38 and MEK signaling pathways. J. Microbiol. Biotechnol., 19, 1355-1363, 2009.

[26] Satoh, A., Tsuji, S., Okada, Y., Murakami, N., Urami, M., Nakagawa, K., Ishikura, M., Katagiri, M., Koga, Y., and Shirasawa, T.: Preliminary clinical evaluation of toxicity and efficacy of a new astaxanthin-rich haematococcus pluvialis extract. J. Clin. Biochem. Nutr., 44, 280-284, 2009.

[27] Hussein, G., Sankawa, U., Goto, H., Matsumoto, K., and Watanabe, H.: Astaxanthin, a carotenoid with potential in human health and nutrition. J. Nat. Prod., 69, 443-449, 2006.

[28] Guerin, M., Huntley, M.E., and Olaizola, M.: Haematococcus astaxanthin: applications for human health and nutrition. Trends Biotechnol., 21, 210-216, 2003.

[29] Richter, C. and Ferrier, J.: Continuously active sodium channels in osteoblastic ROS 17/2.8 cells. Bone Miner., 15, 57-71, 1991.

[30] Bellavite, P.: The superoxide-forming enzymatic system of phagocytes. Free Radic. Biol. Med., 4, 225-261, 1988.

[31] Giulian, D., Vaca, K., and Corpuz, M.: Brain glia release factors with opposing actions upon neuronal survival. $J$. Neurosci., 13, 29-37, 1993.

[32] Lee, S.J., Bai, S.K., Lee, K.S., Namkoong, S., Na, H.J., Ha, K.S., Han, J.A., Yim, S.V., Chang, K., Kwon, Y.G., Lee, S.K., 
and Kim, Y.M.: Astaxanthin inhibits nitric oxide production and inflammatory gene expression by suppressing I(kappa)B kinase-dependent NF-kappaB activation. Mol. Cells, 16, 97105, 2003.

[33] Fox, R.I., Kang, H.I., Ando, D., Abrams, J., and Pisa, E.: Cytokine mRNA expression in salivary gland biopsies of Sjögren's syndrome. J. Immunol., 152, 5532-5539, 1994.

[34] Boumba, D., Skopouli, F.N., and Moutsopoulos, H.M.: Cytokine mRNA expression in the labial salivary gland tissues from patients with primary Sjögren's syndrome. Br. J.
Rheumatol., 34, 326-333, 1995.

[35] Cuello, C., Palladinetti, P., Tedla, N. Di, Girolamo., N., Lloyd, A.R., McCluskey, P.J., and Wakefield, D.: Chemokine expression and leucocyte infiltration in Sjögren's syndrome. Br. J. Rheumatol., 37, 779-783, 1998.

[36] Manabe, E., Handa, O., Naito, Y., Mizushima, K., Akagiri, S., Adachi, S., Takagi, T., Kokura, S., Maoka, T., and Yoshikawa, T.: Astaxanthin protects mesangial cells from hyperglycemia-induced oxidative signaling. J. Cell Biochem., 103, 1925-1937, 2008. 